\title{
Publisher's Note: Photon-momentum transfer in one- and two-photon ionization of atoms [Phys. Rev. A 96, 043414 (2017)]
}

Mu-Xue Wang, Xiang-Ru Xiao, Hao Liang, Si-Ge Chen, and Liang-You Peng (Received 30 October 2017; published 6 November 2017)

DOI: 10.1103/PhysRevA.96.059901

This paper was published online on 17 October 2017 with a typographical error in the author list. The name of the fourth author should read as "Si-Ge Chen." The author's name has been corrected as of 31 October 2017. The author's name is correct in the printed version of the journal. 